\title{
Distribution of static pressure of seeds in a shallow model silo
}

\author{
Józef Horabik* and Marek Molenda \\ Institute of Agrophysics, Polish Academy of Sciences, Doświadczalna 4, 20-290 Lublin, Poland
}

Received September 29, 2016; accepted February 1, 2017

\begin{abstract}
A b s tra c t. The influence of the filling method, the seed size and the aspect ratio on the radial distribution of the vertical pressure on the floor of a shallow model silo and radial distribution of tangent stress within the material was investigated. Three filling methods were applied: central, circumferential and distributed. Seeds of five varieties were used: horse bean, field pea, wheat, vetch, and rapeseed. Vertical pressure on the floor of a shallow bin was found to be influenced by the filling method and the seed size and aspect ratio. A significant dip of vertical pressure in the middle radial location was observed in all tests, except two: central and circumferential filling of rapeseed. Wall friction was mobilized in the highest degree in the case of the central filling, while the lowest was mobilization of friction in the case of the circumferential filling.

K e y w o r d s: bulk of seeds, silo, pressure, friction
\end{abstract}

\section{INTRODUCTION}

One of the first approaches to the mathematical description of the behaviour of granular material in a silo was Janssen article published over 100 years ago (Janssen, 1895). The author proposed a differential equation of the balance of forces on a slice of grain in a silo for evaluation of loads on construction members. Later, static pressure of granular solids in a silo has been extensively investigated experimentally, analytically and numerically (Balevičius et al., 2011; González-Montellano et al., 2012; Lvin, 1971; Nielsen, 1998; Pieper, 1969). Nowadays Janssen approach is recommended as the standard reference method of calculation of the silo pressures (EN 1991-4, 2006). It has been shown that the Janssen approach underestimates the lateral pressure exerted on the wall while the exponential shape

*Corresponding author e-mail: j.horabik@ipan.lublin.pl of pressure distribution agrees with experimental findings (Roberts, 1998). To overcome this discrepancy the Eurocode (EN 1991-4, 2006) recommends using the overpressure factors to raise Janssen pressures to real experimental values. For small depths the profile of wall pressure is nearly linear (Wambaugh et al., 2010) and therefore some design standards (ANSI/ASAE EP446.3, 2013; ANSI/ASAE EP545, 2015) recommend calculating pressures in a shallow silo as the hydrostatic pressure for storage and flowing conditions.

Janssen (1895) assumed that the vertical pressure across the radius of the bin is uniform. Experimental research of Schwab et al. $(1994,1996)$ performed in a full-scale grain silo of $4.1 \mathrm{~m}$ in diameter indicated that the vertical pressure on the silo bottom during filling and detention is not uniform and undergoes some variation. The general tendency is a decrease in pressure with radial distance from a bin center but at the radial location next to the wall a local maximum of pressure was observed. Thompson and Williams (1994) reported that distribution of the vertical pressure on the floor was influenced by the different stiffness of the bin floor in different locations. McBridge (2006) explained this effect by ability of granular solids to transmit shear stress. Pressure measured in locations of greater stiffness than the surrounding area will be over-registered by attracting more load to itself as compared locations with lower stiffness.

In the axially symmetrical state of stress the force balance in the vertical direction for the differential slice of material in cylindrical coordinates $(r, \theta, z)$ is governed by a partial differential equation of local equilibrium (Fig. 1): 


$$
\frac{\partial \sigma_{v}}{\partial z}+\frac{\partial \tau}{\partial r}+\frac{\tau}{r}=\rho g
$$

where: vertical stress $\sigma_{v}(r, z)$ and shear stress $\tau(r, z)$ are functions of radial $r$ and vertical $z$ positions, $\rho$ is bulk density and $g$ is acceleration of gravity. In the approximate approach one can assume that both stresses: $\sigma_{v}$ and $\tau$ are functions of separated variables $z$ and $r: \sigma_{v}=f(r) g(z)$, $\tau=k m \sigma_{v}$, where $k$ is lateral to vertical stress ratio and $\mu$ is the friction coefficient.

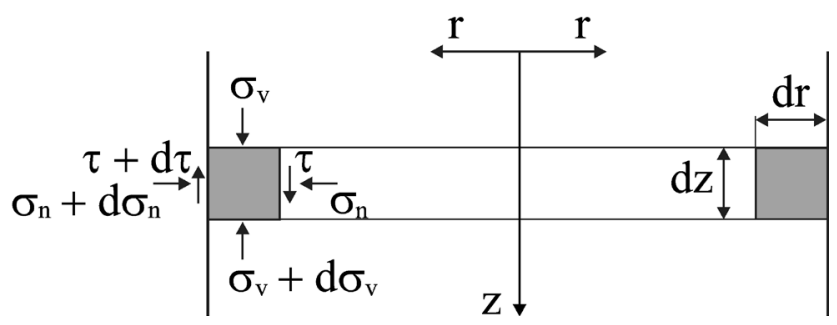

Fig. 1. Differential slice of material in a silo.

Postulating a distribution of $\sigma_{v}$ along the $z$ coordinate and integrating the local equilibrium equation along the $z$ coordinate the partial differential Eq. (1) can be converted into the ordinary differential equation:

$$
\frac{d \tau}{d r}+\frac{\tau}{r}=\rho g-\sigma_{v}^{1}+\sigma_{v}^{0},
$$

where: $\sigma_{v}^{0}$ and $\sigma_{v}^{1}$ is the vertical pressure on integration boundaries $z_{0}$ and $z_{1}$, and $\tau$ is the shear stress averaged along the $z$ coordinate between boundaries $z_{0}$ and $z_{1}$. It can be found from Eq. (2) that for the constant vertical pressure along the radius $\left(\sigma_{v}=\right.$ const.) the shear stress is a linear function of the radial position: $\tau=\mathrm{C} r$, where $\mathrm{C}$ is constant. If the right side of Eq. (2) equals to zero ( $\rho g=\sigma_{v}^{1}-\sigma_{v}^{0}$ ) the shear stress becomes zero along the $r$ coordinate, i.e. the principal stresses are vertical and horizontal. If the vertical pressure is not uniform along the radius, the shear stress changes nonlinearly with the radius.

A number of attempts have been undertaken to omit Janssen assumption that the vertical stress is constant over the horizontal cross section of the silo. Lvin (1971) assumed that the vertical pressure is not constant in a horizontal plane and obtained a family of solutions in which Janssen solution was the lower evaluation of vertical pressure. Similarly, Cowin (1977) derived Janssen type formula of pressure distribution employing weaker assumptions and indicated that Janssen formula gives the lower bound on the vertical and horizontal stresses in the silo.

Possible mechanisms of stress transmission in granular deposit resulting in uneven pressure distribution on the silo floor can be inferred from experimental (Brockbank at al., 1997; McBridge, 2006) and numerical (Zhou and Ooi, 2009; Zhou et al., 2014) studies of loads under a conical stockpile of granular material. Pressure distribution at the base of stockpile forms an ' $\mathrm{M}$ ' shape dip at the center, which can be attributed to the ability of granular solids to transmit static shear stress. McBridge (2006) registered the magnitude of the pressure dip of about $40 \%$ of hydrostatic pressure at the center of $2 \mathrm{~m}$ high stockpile. A significant pressure dip under the apex of pile was confirmed in DEM simulations. The pressure dip under a conical pile composed of non-spherical particles was found more pronounced than under a pile of spherical particles, which produced relatively constant pressure under a pile apex (Zhou and Ooi, 2009; Zhou et al., 2014).

An important factor influencing pressure distribution in the silo is a fabric of the granular assembly which includes particle orientations, the orientation of the contact normal directions and the packing density (Oda, 1978). The fabric may differ in different filling procedures (Horabik et al., 1988; Nielsen, 1983; 1998). An impact of anisotropy of contact orientations on the pressure distribution obtained via DEM simulations was shown by Masson and Martinez (2000) and Sykut et al. (2008) in 2D model silos. The force transmission in bedding of particles and its impact on the pressure distribution in a silo still needs deeper understanding.

The objective of this study was to determine the effects of the filling method, the grain size and the aspect ratio on the radial distribution of the vertical pressure on the floor of a shallow model silo and the radial distribution of the shear stress within the material.

\section{METHODS AND MATERIALS}

Experiments were conducted to determine the effect of the filling method on the radial distribution of vertical pressure on the bottom of a model bin. Height to diameter ratio of the model bin corresponds to shape of agricultural grain silos and shallow bins (ANSI/ASAE EP545, 2015; Wojnowski and Bernhardt, 2015). A schematic diagram of the experimental apparatus is shown in Fig. 2a. The bin was $0.61 \mathrm{~m}$ in diameter and $0.62 \mathrm{~m}$ high. The two semicircular halves were connected with four load cells installed in pairs on the two connection lines, restoring the cylindrical shape of the wall. The silo wall, constructed of galvanized steel $3 \mathrm{~mm}$ thick, was $0.6 \mathrm{~m}$ in diameter and $0.6 \mathrm{~m}$ high. The flat bottom was divided into five concentric rings of equal surface area $(i=1, \ldots, 5)$. Each of the rings and the wall was supported on three load cells spread at an angular distance of $120^{\circ}$ apart $(j=1,2$, and 3). Loads were measured with an accuracy of $\pm 0.5 \mathrm{~N}$ through a data acquisition system. The experimental set-up allowed for the determination of the distribution of vertical pressure on the bottom $\sigma_{v}$ between 5 rings, mean lateral pressure $\sigma_{n}$, and shear stress $\tau$ on the wall (Molenda et al., 1996a). The apparent coefficient of wall friction $\mu^{*}$ was determined as the ratio of mean shear stress $\tau$ to mean lateral pressure $\sigma_{n}$. Applying simplified recommendation of standards on pressures calculations in shallow silo (ANSI/ASAE EP446.3, 2013; 
a

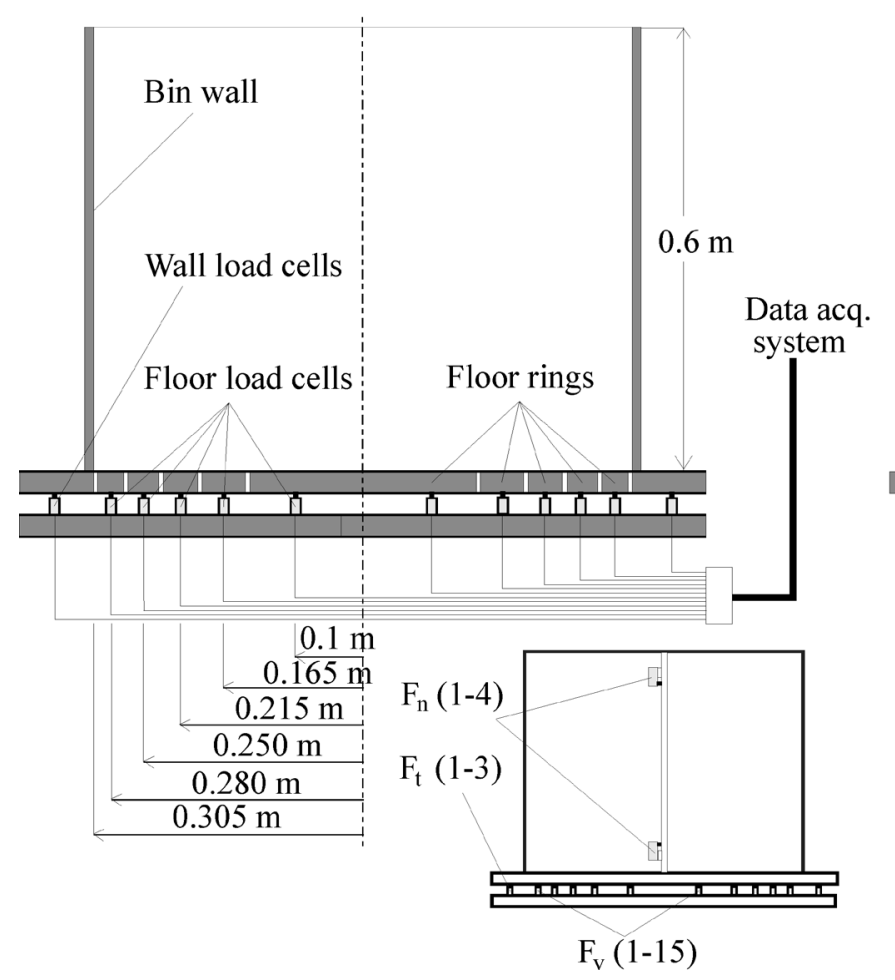

b

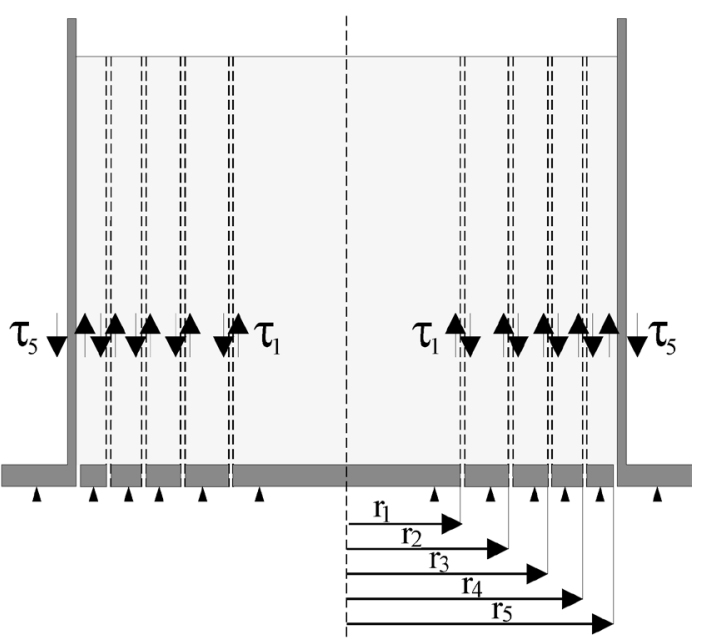

Fig. 2. Experimental set: a - details of the model bin construction, b - imaginary cylindrical slices of the granular material in the bin.

ANSI/ASAE EP545, 2015) the average value of pressure ratio $k$ was determined as ratio of the mean lateral pressure $\sigma_{n}$ to the vertical pressure averaged along the height of the seed deposit, which equals to 0.5 of the pressure on the silo bottom $\sigma_{v}$ :

$$
k=\frac{2 \sigma_{n}}{\sigma_{v}} .
$$

Assuming that bulk density of particle bedding is constant along the bin radius, averaged shear stress $t_{i}(i=1, \ldots$, $5)$ on the imaginary cylindrical cross-sections of the grain bedding separating material over $i$-th ring of the silo bottom from material over $i+1$-th ring (Fig. $2 \mathrm{~b}$ ) was calculated from the balance of forces in the vertical direction on the finite dimension cylindrical slice of the material following the Eq. (2):

$$
\tau_{i}=\frac{i \pi r_{5}^{2} h \rho g / 5-\sum_{1}^{i} \sum_{j=1}^{3} F_{v_{-} i, j}}{2 \pi r_{i} h},
$$

where: $F_{v i, j}$ is the force recorded by load cell $i, j$ supporting ring $i(i=1, \ldots, 5)$ on cell $j(j=1,2$, and 3$)$.

To explore an impact of the packing structure on the load distribution at the bottom and the wall of a shallow bin, three filling methods were applied, as follows: central, circumferential and distributed. The filling device was
Central

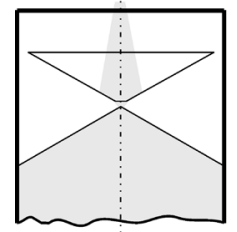

Fig. 3. Filling methods.

gradually moved up during the filling process to reduce the effect of kinetic energy on the bedding structure of the pile (Fig. 3).

Seeds of five varieties of cultivated plants at storage moisture content were used as the granular material in the study: horse bean ( $c v$. Nadwiślański), field pea ( $c v$. Szelejewska), vetch ( $c v$. Helia), wheat ( $c v$. Eta) and rapeseed ( $c v$. Licosmos). Basic geometrical parameters of seed and mechanical parameters of seed in bulk are collected in Table 1. Measurements of three major perpendicular dimensions, namely length $(a)$, width $(b)$, and thickness $(c)$ of the seed were carried out using a manual caliper with an accuracy of $0.01 \mathrm{~mm}$ for randomly selected samples of 50 seeds. The equivalent diameter was determined as the geometric mean of three dimensions $a, b$, and $c$ : 


$$
d=\sqrt[3]{a b c}
$$

and aspect ratio $\alpha$ was determined as the ratio of the length to the thickness:

$$
\alpha=\frac{a}{c} .
$$

The true density and the porosity of $100 \mathrm{~cm}^{3}$ samples of seed were determined by measuring the weight of samples and its true volume using an air pressure pycnometer (Mohsenin, 1986). The angle of the internal friction was determined in a Jenike shear tester $(21 \mathrm{~cm}$ in diameter $)$ T a b l e 1. Physical parameters of seed according to the procedure recommended by EN 1991-4 (2006). Samples of seed pre-loaded to pressure of $55 \mathrm{kPa}$ were sheared at 5 levels of normal pressure ranging from 14 to $55 \mathrm{kPa}$ with the displacement velocity of $1.3 \mathrm{~mm} \mathrm{~s}^{-1}$. Each test was repeated three times.

\section{RESULTS}

The radial distribution of the vertical pressure on the bottom of the bin and the shear stress in bulk of seed was found to be influenced by the filling method and the seed size and shape (Figs 4 and 5). Most of differences are statistically significant at the significance level of 0.05 . Bars

\begin{tabular}{lcccccc}
\hline Seed & $\begin{array}{c}\text { Moisture } \\
\text { content }(\%)\end{array}$ & $\begin{array}{c}\text { Equivalent } \\
\text { diameter, } d \\
(\mathrm{~mm})\end{array}$ & Aspect ratio, $\alpha$ & $\begin{array}{c}\text { True density, } \rho \\
\left(\mathrm{kg} \mathrm{m}^{-3}\right)\end{array}$ & $\begin{array}{c}\text { Angle of } \\
\text { Porosity }(\%)\end{array}$ & $\begin{array}{c}\text { Anternal friction, } \\
\varphi\left({ }^{\circ}\right)\end{array}$ \\
\hline Horse bean & 11.7 & $8.22 \pm 0.25$ & $1.48 \pm 0.03$ & $1337 \pm 7$ & $32.7 \pm 0.7$ & $29.3 \pm 0.6$ \\
Field pea & 11.8 & $6.11 \pm 0.15$ & $1.22 \pm 0.03$ & $1219 \pm 6$ & $30.7 \pm 1.4$ & $28.8 \pm 0.5$ \\
Vetach & 12.7 & $4.14 \pm 0.12$ & $1.28 \pm 0.03$ & $1214 \pm 6$ & $30.2 \pm 0.5$ & $22.1 \pm 0.6$ \\
Wheat & 12.8 & $3.96 \pm 0.09$ & $1.9 \pm 0.03$ & $1252 \pm 6$ & $36.2 \pm 0.1$ & $24.6 \pm 0.8$ \\
Rapeseed & 6.8 & $1.86 \pm 0.06$ & $1.31 \pm 0.03$ & $1022 \pm 5$ & $31.5 \pm 0.7$ & $24.3 \pm 0.7$ \\
\hline
\end{tabular}
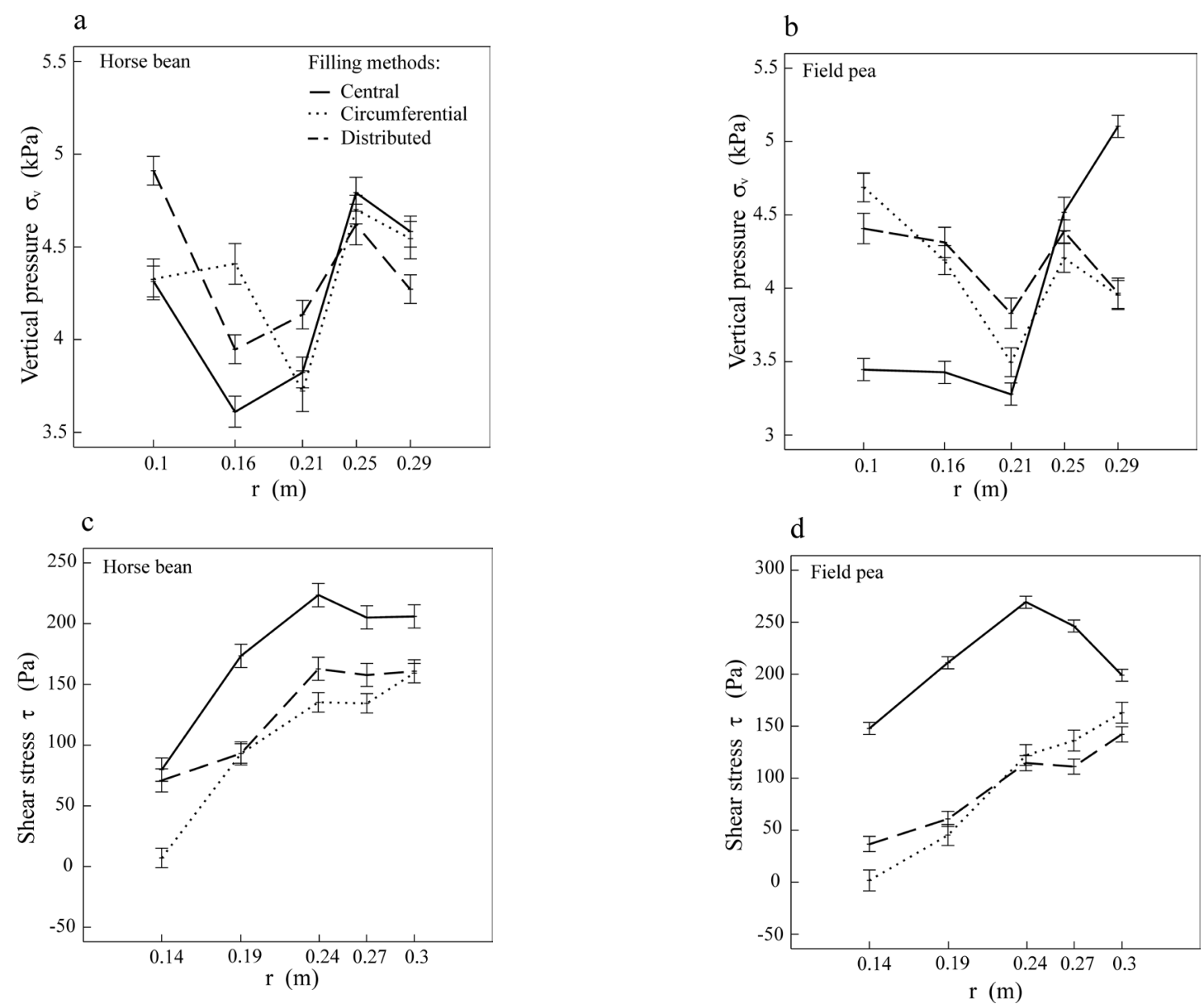

Fig. 4. Radial distribution of vertical pressure $\sigma_{n}$ on silo bottom of horse bean (a) and field pea (b) and approximated shear stress $\tau$ in bulk of horse bean (c) and field pea (d) as affected by the filling method. 

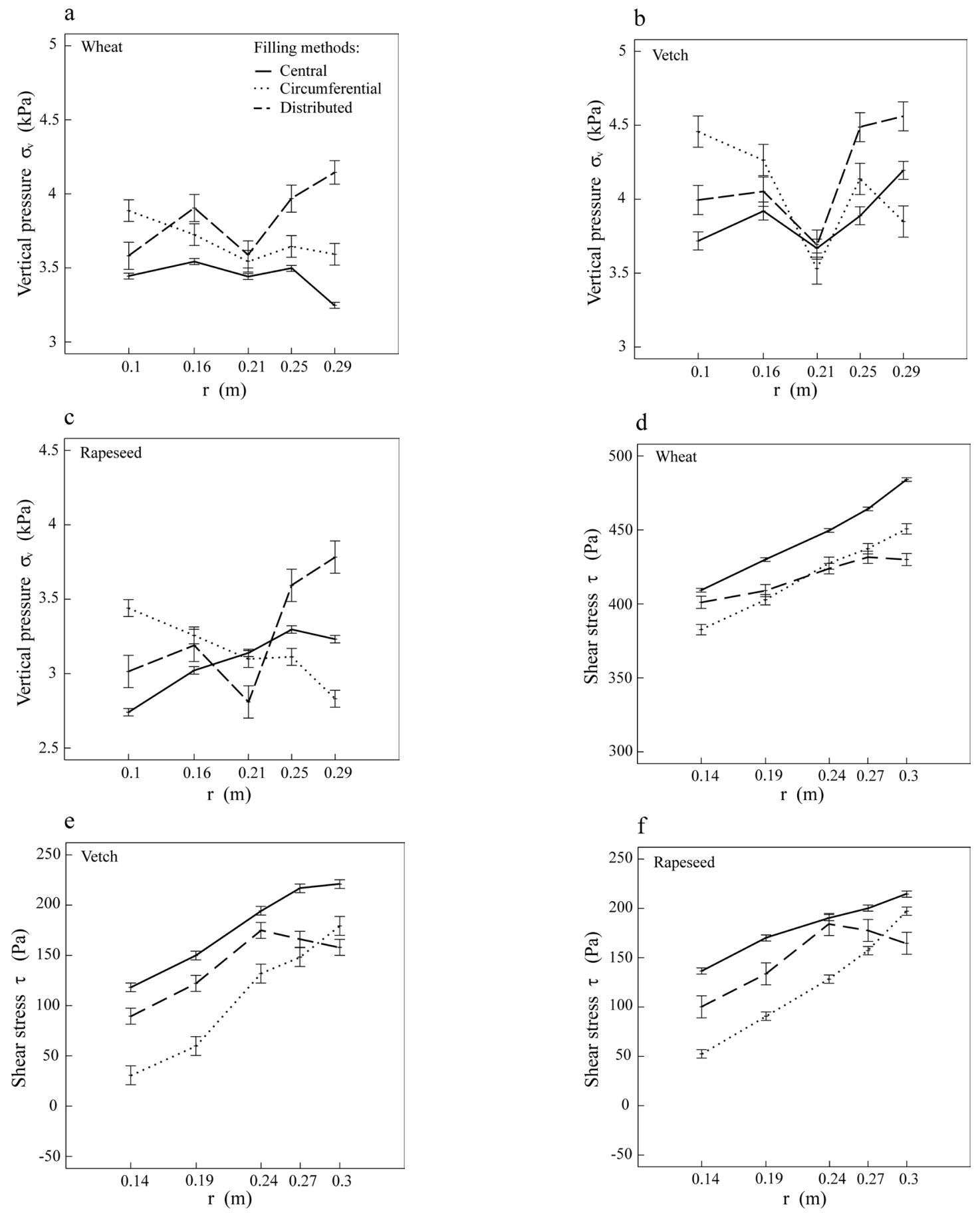

Fig. 5. Radial distribution of vertical pressure on silo bottom $\sigma_{n}$ of wheat (a), vetch (b) and rapeseed (c) and approximated shear stress $\tau$ in bulk of wheat (d), vetch (e) and rapeseed (f) as affected by the filling method.

in Figs 4 and 5 denote 95\% confidence intervals. Note that different positions along the $r$ axis are used to present the vertical stress and the shear stress. The vertical stress (Figs 4a,b and 5a,b,c) refers for radial locations of load cells supporting bottom rings (Fig. 2a), while the shear stress (Figs 4c,d and 5d,e,f) refers to radius of the cylinder separating material between neighboring rings (Fig. 2b). A significant dip of vertical pressure in a middle radial loca- tion was observed in all cases of experiment, except two: the central and circumferential filling of rapeseed, i.e. the smallest seed. In the case of rapeseed a very clear trend of decrease of the vertical pressure for the circumferential filling and the pressure increase for the central filling with radial distance to the bin center were observed. In the case of bigger seeds the vertical pressure distributions revealed summing-up effect of two components: 
T a b l e 2. Mechanical parameters of bulk of seed determined in the model silo

\begin{tabular}{lccccc}
\hline Seeds & Filling methods & $\begin{array}{c}\text { Bulk density, } \\
\rho\left(\mathrm{kg} \mathrm{m}^{-3}\right)\end{array}$ & Porosity (\%) & $\begin{array}{c}\text { Apparent } \\
\text { coefficient of wall } \\
\text { friction, } \mu^{*}\end{array}$ & $\begin{array}{c}\text { Pressure ratio, } k \\
\text { Horse bean }\end{array}$ \\
Field pea & central & $900 \pm 4$ & $32.7 \pm 0.4$ & $0.232 \pm 0.014$ & $0.510 \pm 0.024$ \\
Wheat & central & $845 \pm 3$ & $30.7 \pm 0.4$ & $0.244 \pm 0.012$ & $0.503 \pm 0.020$ \\
Wheat & central & $799 \pm 4$ & $36.2 \pm 0.4$ & $0.313 \pm 0.004$ & $0.409 \pm 0.005$ \\
Wheat & circumferential & $760 \pm 4$ & $39.3 \pm 0.4$ & $0.286 \pm 0.002$ & $0.380 \pm 0.003$ \\
Vetach & central & $800 \pm 4$ & $36.1 \pm 0.4$ & $0.303 \pm 0.007$ & $0.301 \pm 0.007$ \\
Rapeseed & central & $700 \pm 2$ & $30.2 \pm 0.4$ & $0.195 \pm 0.005$ & $0.672 \pm 0.015$ \\
\hline
\end{tabular}

- global: similar to the case of the rapeseed trend of the vertical pressure changes with the radial position for the central and circumferential filling; and

- local: a strong pressure dip in the center of the bin radius. As the consequence in course of the resultant pressure distribution one can distinguish the highest value of the vertical pressure in the location closest to the bin center for the circumferential filling and in the location closest to the wall for the central filling, and a clearly visible pressure dip in the middle radial location.

The distributed filling generated a dip in the vertical pressure distribution for all materials. Except the rapeseed, the vertical pressure distribution was similar to that produced by the circumferential filling, while for rapeseed the pressure distribution was closer to that produced by the central filling. With the distributed filling the top layer of material was horizontal, which gave particles the most favourable conditions of laying with the longest axis oriented horizontally as compared to two other filling methods.

The shear stress is directly related to the vertical pressure according to Eq. (1). It means that the distribution of the averaged shear stress on cylindrical cross-sections of the bulk of seed is a direct consequence of a vertical pressure distribution on the silo bottom. Any departure in the vertical pressure on the silo bottom from the uniform distribution results in the departure of the shear stress from a linear distribution. In most cases the shear stress distribution increased almost linearly with the radial distance to the silo center (Figs 4c,d and 5d,e,f). The increase was smooth and almost linear in the cases of the relatively low dip in vertical pressure $\sigma_{v}$ (circumferential filling, all materials), while in the cases of the high dip in vertical pressure $\sigma_{v}$ (central filling: horse bean, filed pea; distributed filling: horse bean, vetch, rapeseed) the shear stress increased to the maximal value at the radial location of about 0.8 of the bin radius and then decreased with increasing proximity to the wall. The shear stress was the lowest in the case of the circumferential filling and the highest in the case of the central filling. It means that from the applied filling methods, the circumferential filling produces the pressure distribution involving the particle-particle friction in the smallest degree, i.e. it generates the pressure distribution akin to a hydrostatic state. Following distributions of the shear stress, the apparent coefficient of wall friction $\mu^{*}$ was also mobilized to the highest degree in the case of the central filling and to the lowest degree in the case of the circumferential filling (Table 2).

The average value of pressure ratio $k$ calculated by means of numerical solution of Janssen Eq. (2) for the mean value of vertical pressure on bottom and the mean value of mobilization of wall friction coefficient $\mu^{*}$ (Molenda et al., 1996b) was found to be the highest for vetch and the lowest for wheat (Table 2). This finding corresponds well with the lowest value of the internal friction angle of vetch seed providing the highest value of the pressure ratio calculated according to the simplified formula recommended by EN 1991-4 (2006), $k=1.1(1-\sin \varphi)$. The reason for the lowest value for wheat seems to be the most non-spherical shape of grain.

Bulk density was found to be almost non-sensitive to the filling method, except the case of wheat grain, for which the bulk density in the case of the circumferential filling was $5 \%$ lower than in the central and distributed filling (Table 2).

\section{DISCUSSION}

Local minimum in distribution of the vertical pressure, approximately in the center of the bin radius, may be explained by the ability of granular solids to form long force chains transmitting stress to distant. The second factor influencing the pressure distribution is the fabric of the granular assembly which may differ in different filling methods (Horabik et al., 1988; Nielsen, 1983). A gentle filling of the silo from an orifice that was slowly raised resulted in a preferred orientation of the contact directions that followed the line of the generatrix of the cone of 
natural repose of the particles (Horabik et al., 2016). The central filling produces the preferred orientation of the contact directions along the downward oriented generatrix line of the cone while the circumferential filling results in the upward oriented generatrix line. The preferred orientation of the contact directions enforces a transmission of the load outside the location of the filling device through long-range force chains. That results in the highest value of the vertical pressure on the bottom close to the wall for the central filling and close to the bin center for the circumferential filling. Preferred orientation of particles may influence also bulk density of material. Summation of impact of the highest non-spherical shape of wheat grain $(\alpha=1.9)$ with strong anisotropy of grain orientation generated by down oriented apex of natural repose cone may be responsible for bulk density of wheat grain lower for the case of the circumferential filling as compared to the central filling. Lower value of the pressure ratio and mobilization of the wall friction in the case of the circumferential filling as compared to the central one suggest that circumferential filling of nonspherical particles generates looser structure transmitting more load into bottom.

As observed in this study, the increase in the shear stress with increasing distance from the bin axis, accompanied or not by a maximum in the wall vicinity supports Drescher (1991) hypothesis of yielding of granular solids at the wall or in the wall vicinity. This assumption was applied for the proposition of the analytical method of the determination of the pressure ratio using two material parameters: mobilization of wall and internal friction and assumed localization of yielding of material. The obtained distributions of shear stress in bulk of seed support hypothesis that the maximum degree of mobilization of the internal friction occurs in the vicinity of the wall or exactly in the particle-wall contact.

According to Cowin (1977) Janssen constant should be interpreted as the ratio of the horizontal stress averaged over the lateral boundary perimeter to the vertical stress averaged over the cross-sectional area rather than as the ratio of local horizontal to vertical pressure. An important implication of Cowin analysis was that it was not possible to construct lower bound on $\sigma_{v}$ unless at least one of the principal stresses in the plane of the cross section varies over some part of the cross section and, over the same part of the cross section there is a function of vertical coordinate. The results reported in this study support Cowin conclusion that Janssen constant should be interpreted as the ratio of the averaged horizontal and lateral stresses.

\section{CONCLUSIONS}

1. Vertical pressure on the bottom of a shallow bin was found to be influenced by the filling method, the seed size and the aspect ratio. A significant dip in vertical pressure close to the halfway point of the silo radius was observed in all cases of experiment, except two: the central and circumferential filling of rapeseed.
2. Generally the shear stress in bulk of seed increased almost linearly with radial distance to the silo center. In the cases of the highest dip in vertical pressure the shear stress increased to the maximum at the radial location of about 0.8 of the bin radius and then decreased with increasing proximity to the wall.

3. Wall friction was mobilized in the highest degree in the case of the central filling and in the lowest degree in the case of the circumferential filling.

Conflict of interest: The Authors do not declare conflict of interest.

\section{REFERENCES}

ANSI/ASAE EP446.3, 2013. Loads exerted by Irish potatoes in shallow bulk storage structures. American Society of Agricultural and Biological Engineers, St. Joseph, Mi, USA.

ANSI/ASAE EP545, 2015. Loads exerted by free-lowing grain on shallow storage structures. American Society of Agricultural and Biological Engineers, St. Joseph, Mi, USA.

Balevičius R., Sielamowicz I., Mróz Z., and Kačianauskas R., 2011. Investigation of wall stress and outflow rate in a flatbottomed bin: A comparison of the DEM model results with the experimental measurements. Powder Technol., 214, 322-336.

Brockbank R., Huntley J.M., and Ball R.C., 1977. Contact force distribution beneath a tree-dimensional granular pile. J. Phys. II France, 7, 1521-1532.

Cowin S.C., 1977. The theory of static loads in bins. J. Appl. Mech., 44(3), 409-412.

Drescher A., 1991. Analytical methods in bin-load analysis. Elsevier, Amsterdam-Oxford-New York-Tokyo.

EN 1991-4, 2006. Eurocode 1. Basis of design and actions on structures e Part 4: Actions in silos and tanks. Rue de Stassart 36, B-1050 Brussels: European Committee for Standardization. Central Secretariat.

González-Montellano C., Gallego E., Ramírez-Gómez Á., and Ayuga F., 2012. Three dimensional discrete element models for simulating the filling and emptying of silos: Analysis of numerical results. Comput. Chem. Eng., 40, 22-32.

Horabik J., Parafiniuk P., and Molenda M., 2016. Experiments and discrete element method simulations of distribution of static load of grain bedding at bottom of shallow model silo. Biosyst. Eng., 149, 60-71.

Horabik J.A., Ross I.J., and Schwab C.V., 1988. Effects of spatial orientation on grain load distribution. Trans. ASAE, 31(6), 1787-1793.

Janssen H.A., 1895. Versuche über Getreidedruck in Silozellen. Verein Deutscher Ingenieure, Zeitschrift (Düsseldorf), 39, 1045-1049.

Liffman K., Nguyen M., Metcalfe G., and Cleary P., 2001. Forces in piles of granular material: An analytic and 3D DEM study. Granul. Matter, 3(3), 165-176.

Lvin J.B., 1971. Analytical evaluation of pressures of granular materials on silo walls. Powder Technol., 4, 280-285.

Masson S. and Martinez J., 2000. Effect of particle mechanical properties on silo flow and stress from distinct element simulations. Powder Technol., 109, 164-178. 
McBridge W., 2006. Base pressure measurements under a scale model stockpile. Particul. Sci. Technol., 24, 59-70.

Mohsenin N.N., 1986. Physical properties of plant and animal materials. New York, Gordon and Breach Science Press, New York, USA.

Molenda M., Horabik J., and Ross I.J., 1996a. Effect of filling method on load distribution in model grain bins. Trans. ASAE, 39(1), 219-224.

Molenda M., Horabik J., and Ross I.J., 1996b. Pressure distribution and stress ratio in a model grain silo. XIII CIGR World Congress, Madrid, Spain, Paper 96B-008.

Nielsen J., 1983. Load distributions in silos influenced by anisotropic grain behaviour. Int. Conf. Bulk Materials, Storage, Handling and Transportation, Newcastle, NSW, Australia, August 22-24, The Institution of Engineers, Australia.

Nielsen J., 1998. Pressures from flowing granular solids in silos. Phil. Trans. R. Soc. A, 356(1747), 2667-2684.

Oda M., 1978. Significance of fabric in granular mechanics. Proc. U.S.-Japan Seminar Continuum Mechanical and Statistical Approaches in the Mechanics of Granular Materials, June 5-9, Tokyo, Japan.

Pieper K., 1969. Investigation of silo loads in measuring models. J. Eng. Industry. Trans. ASME, 91, 365-372.

Roberts A.W., 1998. Particle technology - Reflections and horizons: An engineering perspective. Trans. IChemE, 76, Part A, 755-796.
Schwab C.V., Ross I.J., White G.M., and Colliver D.G., 1994. Wheat loads and vertical pressure distribution in a fullscale bin. 1. Filling. Trans. ASAE, 37(5), 1613-1619.

Schwab C.V., Ross I.J., White G.M., and Colliver D.G., 1996. Wheat loads and vertical pressure distribution in a fullscale bin. 2. Detention. Trans. ASAE, 39(3), 1145-1149.

Sykut J., Molenda M., and Horabik J., 2008. Influence of filling method on packing structure in model silo and DEM simulations. Granular Matter, 10, 273-278.

Thompson S.A. and Williams R.A., 1994. The effect of floor flexibility on the vertical pressures in a model grain bin. Trans. ASAE, 37(2), 623-628.

Wambaugh J.F., Harley R.R., and Behringer R.P., 2010. Force networks and elasticity in granular silos. Eur. Phys. J. E, 32(2), 135-145.

Wojnowski D.A. Bernhardt R., 2015. Analysis of Design Standards and Recommended Practices for Agricultural Storage Bins. Forensic Engineering 2015. Performance of the Built Environment, 756-765.

Zhou Ch. and Ooi J.Y., 2009. Numerical investigation of progressive development of granular pile with spherical and non-spherical particles. Mech. Mater., 41, 707-714.

Zhou Z.Y., Zou R.P., Pinson D., and Yu A.B., 2014. Angle of repose and stress distribution of sandpiles formed with ellipsoidal particles. Granular Matter, 16, 695-709. 